\title{
Cost-Effectiveness of Point-of-Care C-Reactive Protein Tests for Respiratory Tract Infection in Primary Care in England
}

Rachael Hunter

To view enhanced content go to www.advancesintherapy.com Received: October 29, 2014 / Published online: January 27, 2015

(c) The Author(s) 2015. This article is published with open access at Springerlink.com

\section{ABSTRACT}

Introduction: Despite recommendations that general practitioners (GPs) delay antibiotic prescribing for respiratory tract infections (RTIs), antibiotic prescriptions in primary care in England increased by $4.1 \%$ from 2010 to 2013. C-reactive protein (CRP) point-of-care tests (POCT), for example, the Afinion $^{\mathrm{TM}}$ Analyzer (Alere Ltd, Stockport, UK) device, are widely used in several countries in the European Union. Studies suggest that CRP POCT use, either alone or in combination with communication training, reduces antibiotic prescribing and improves quality of life for patients presenting with RTI symptoms. The aim of this study is to evaluate the costeffectiveness of CRP POCT for RTIs in primary care in England over 3 years for three different strategies of care compared to standard practice.

Electronic supplementary material The online version of this article (doi:10.1007/s12325-015-0180-x) contains supplementary material, which is available to authorized users.

R. Hunter $(\bowtie)$

Research Department of Primary Care and

Population Health, Royal Free Medical School,

London NW3 2PF, UK

e-mail: r.hunter@ucl.ac.uk
Methods: An economic evaluation was carried out to compare the costs and benefits of three different strategies of CRP testing (GP plus CRP; practice nurse plus CRP; and GP plus CRP and communication training) for patients with RTI symptoms as defined by National Institute for Health and Care Excellence guideline CG69, compared with current standard GP practice without CRP testing. Analysis consisted of a decision tree and Markov model to describe the quality-adjusted life years (QALYs) and cost per 100 patients, together with the number of antibiotic prescriptions and RTIs for each group.

Results: Compared with current standard practice, the GP plus CRP and practice nurse plus CRP test strategies result in increased QALYs and reduced costs, while the GP plus CRP testing and communication training strategy is associated with increased costs and reduced QALYs. Additionally, all three CRP arms led to fewer antibiotic prescriptions and infections over 3 years.

Conclusion: The additional cost per patient of the CRP test is outweighed by the associated cost savings and QALY increment associated with a reduction in infections in the long term. 
Keywords: Antibiotics; Cost-effectiveness; C-reactive protein; CRP test; Primary care; Quality-adjusted life years; Respiratory tract infection

\section{INTRODUCTION}

In England, clinical guidance from the National Institute for Health and Care Excellence (NICE) on respiratory tract infection (RTI) recommends that general practitioners (GPs) delay prescribing of antibiotics and advise patients that antibiotics may have no impact on symptoms in suspected viral infections [1]. Despite this recommendation, antibiotic prescribing increased by $4.1 \%$ between 2007 and 2011 in England [2]. As most of these infections are likely to be viral and current evidence suggests that antibiotic prescribing confers no additional benefit [3], it is likely that most of the prescribing is unnecessary and increases the risk of antibiotic-resistant infections. The Department of Health has developed a 5-year strategy running from 2013 to 2018 in England which aims to slow the development and spread of antimicrobial resistance, by prescribing antibiotics only to patients who are likely to benefit [4].

C-reactive protein (CRP) point-of-care tests (POCT) are widely used in many European Union (EU) countries for antibiotic prescription decision-making, and a metaanalysis found that they significantly reduce antibiotic prescribing compared to usual care [5]. Other studies have provided evidence that communication training for health-care professionals can be an effective way to reduce the prescription of antibiotics to patients with RTIs $[6,7]$.

Cost-effectiveness analyses conducted previously have shown that although CRP tests cost more per patient in the short term (€11.27 per patient in Oppong et al. [8]), this is balanced by a reduction in antibiotic prescriptions $(€ 112.70$ per prescription avoided) and improved quality of life [8]. Evidence of the cost-effectiveness of CRP tests for RTIs versus current practice is limited to analyses alongside trials, none of which look at costs and outcomes beyond 28 days or from the National Health Service (NHS) England perspective.

The aim of this study is to present the results of a decision analytic model of the costeffectiveness of near-patient CRP tests for RTI in NHS England over 3 years.

\section{METHODS}

\section{Basic Characteristics of Analysis}

The model compares the current standard in GP practice, where patients presenting with RTI symptoms are prescribed antibiotics dependent on GPs' views and patient expectations (hereafter called current practice), and the three different strategies of CRP testing for patients presenting with RTI symptoms:

i. GP plus CRP: The patient is tested by the GP using a point-of-care CRP test and prescribed antibiotics accordingly.

ii. Practice nurse plus CRP: The patient is tested by the primary care nurse using a point-of-care CRP test and the results passed onto the GP who prescribes antibiotics accordingly. This strategy has been included as a comparator since it is a potential patient pathway considered by GP practices who feel that it might be more reasonable to delegate this responsibility to the practice nurse given the time implications of the CRP test. 
iii. GP plus CRP and communication training: The strategy is the same as (i) GP plus CRP, except that the GP also receives training on communicating with patients about RTI and antibiotics.

It is assumed that, for all three strategies with CRP, the GP receives training on how to use CRP as an aid to diagnosis and how to use the CRP test. Additionally, in strategy (ii) the practice nurse also receives training on CRP.

\section{Model Structure}

The economic evaluation consists of a decision tree (Fig. 1) and Markov model (Fig. 2) and was developed in Excel 2010 (Microsoft Corporation, Redmond, WA, USA). The structure for the decision tree is based on a similar model developed by NICE [1]. A Markov model was chosen for the movement between health states as it is the most appropriate way to calculate movements between health states when many movements are required.

For all patients the decision tree progresses as follows:

- current practice or one of the three CRP arms;

- antibiotic prescription;

- if prescribed antibiotics, the risk of antibiotic-related adverse events including death;

- complications such as otitis media, quinsy, or sinusitis;

- prescription of antibiotics within 28 days of index consultation;

- complications and adverse events from antibiotic prescriptions within 28 days of index consultation.

At baseline, cohorts of 100 hypothetical patients with RTI (with assumed characteristics of adult patients that attend primary care with RTI symptoms, i.e., 50 years old, $62 \%$ female
[9]) enter each arm of the four strategies and receive either one of the three CRP test strategies or current practice (no CRP test). They then enter the decision tree (Fig. 1). The probability that a patient is then prescribed antibiotics is dependent on the CRP test or current practice arm of the model they are in. The probabilities are taken from trial data, but it is likely that prescribing in the CRP test arms is influenced by the results of the CRP test.

It is assumed that patients who are prescribed antibiotics then go on to take them. Antibiotics carry with them a risk of adverse reactions including anaphylactic reaction to penicillin, which carries a risk of death. Not prescribing antibiotics carries the risk of complications including sinusitis, otitis media and quinsy. Antibiotic prescription carries a risk of similar complications, but the risk is lower [1].

The probability that patients have been prescribed antibiotics within 28 days of baseline (the index consultation) has been included in the model along with the costs and complications associated with antibiotic prescription for patients prescribed antibiotics.

The Markov model has only two health states: healthy and RTI. The probability of death is only contained in the decision tree and occurs only for patients who have an adverse event following antibiotic prescription. Of the two health states in the Markov model, RTI is the only state that contains costs, since it requires a response from the health system. Patients that enter the RTI health state are only those that attend primary care as a result of RTI symptoms. The decision tree in Fig. 1 is also used in the RTI health state to calculate the probability of antibiotic prescription, antibioticrelated adverse events including death, complications associated with prescribing or delaying antibiotics, and the probability that 


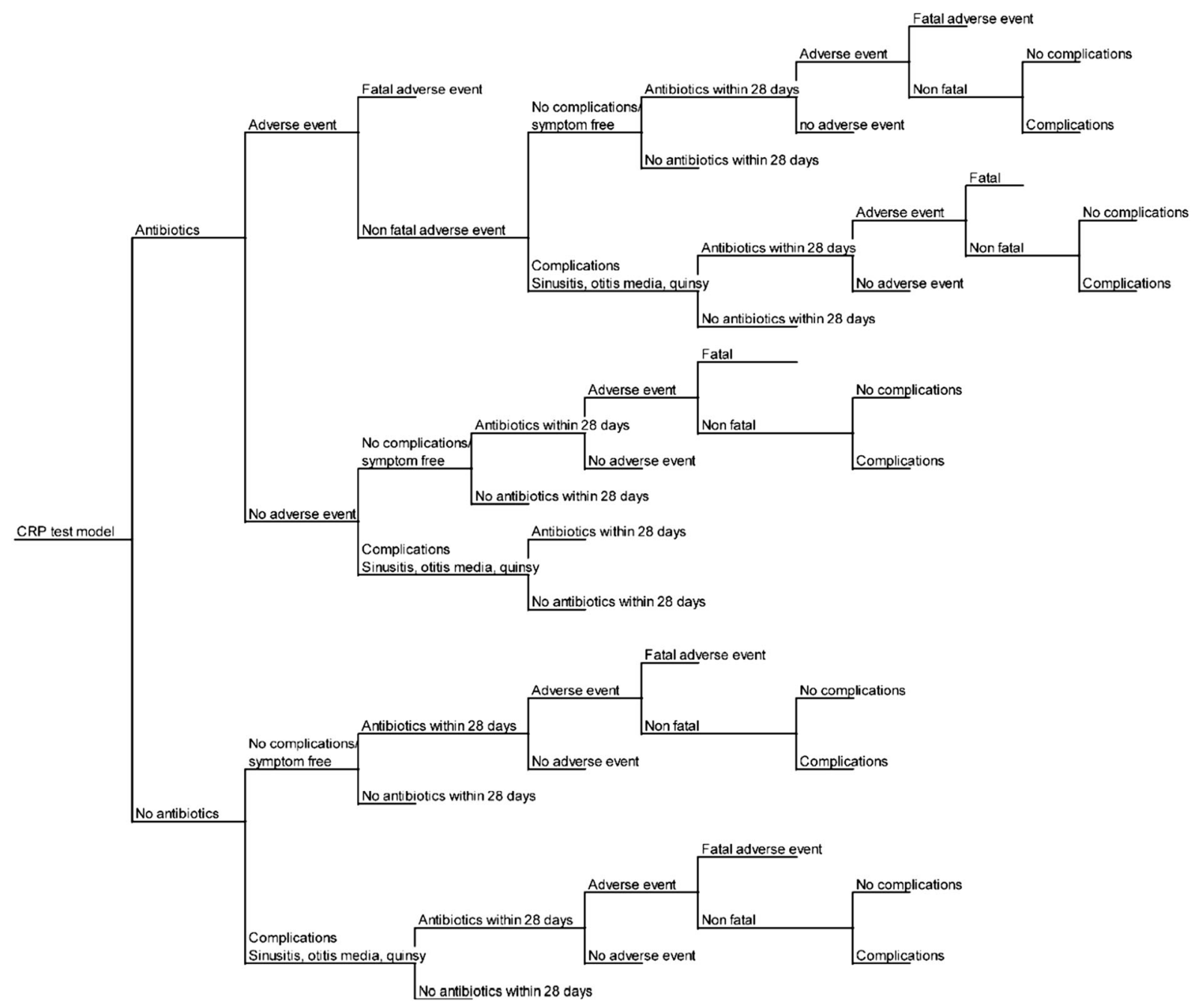

Fig. 1 Decision tree for current practice and the three CRP test strategies. Decision-making criteria used to evaluate the cost-effectiveness of CRP testing and current practice. CRP C-reactive protein

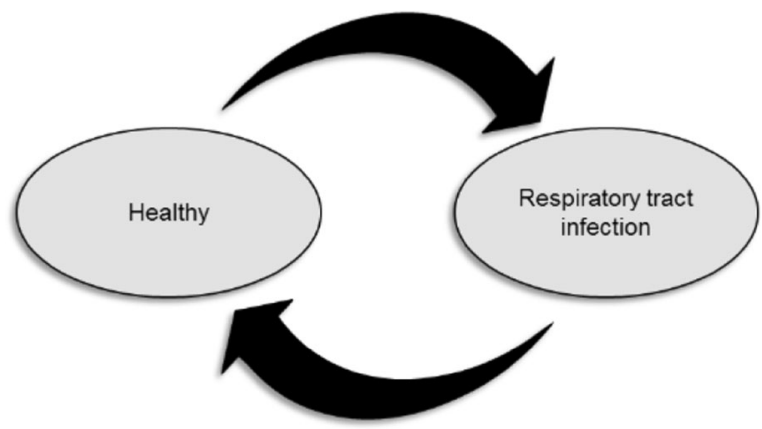

Fig. 2 Markov model for current practice and the three C-reactive protein test strategies. The Markov model depicts two states: healthy and respiratory tract infection the patient is prescribed antibiotics within 28 days of the index consultation for this episode of RTI. The probability of being prescribed antibiotics for an RTI after the first cycle in the model (first 28 days) is based on the results of the 3-year study by Cals et al. [9] to reflect the observed change in GP behavior following the index consultation and 28 days follow-up. The two states have health state-specific outcomes, expressed as utility scores used to calculate quality-adjusted life years (QALYs). 
As stated above, the cycle length is 28 days and the total time horizon is 3 years ( 40 cycles). Three years has been chosen for the duration of the model to reflect the results from the recently published paper by Cals et al. [9] There is no data available on GP antibiotic prescribing and RTI infections following the implementation of CRP for a time horizon greater than 3 years.

\section{Probabilities: Decision Tree}

The probabilities used to populate the decision tree are listed in Table 1 [1, 5-7, 9-11]. For the current practice and GP plus CRP arms of the model, the probability of patients presenting with RTI being prescribed antibiotics at the index consultation (baseline) was taken from a systematic review [5]. The results of Little et al. [7] have been added to these, since they were published too late to be included in the systematic review. The probability of antibiotic prescription for practice nurse plus CRP was derived from Cals et al. [10] as that is the pointof-care CRP test protocol used in this study. Antibiotic prescription at the index consultation for the GP plus CRP and communication training strategy was taken from Cals et al. [10] and Little et al. [7] using the numbers reported in the paper to calculate the percentage of patients prescribed antibiotics.

In the decision tree, patients prescribed antibiotics have a small percentage (0.05\%) chance of an adverse event, anaphylactic shock [11]. Patients that have this adverse event have a $10 \%$ chance of death [1]. All patients have a percentage chance of complications including sinusitis, otitis media, or quinsy, although the chance of complications is dependent on being prescribed antibiotics at the initial consultation.

All patients also have a probability of being prescribed antibiotics within 28 days of the index consultation, dependent on the arm of the model (current practice or one of the CRP arms). The probability of being prescribed antibiotics within 28 days of the index consultation has been taken from the same studies as baseline (the index consultation) antibiotic prescription, except that no 28-day values were available for Little et al. [7]. Patients prescribed antibiotics within 28 days of the initial consultation also have a percentage chance of antibiotic-related adverse events and complications with the same values as being prescribed antibiotics at baseline.

The same decision tree is also used for patients with incidents of RTI in the subsequent cycles of the Markov model following the first 28 days. The only difference between the first decision tree and decision trees for subsequent incidents of RTI is the probability that patients are prescribed antibiotics when they first attend the GP for this incident of the RTI. The probability that patients are prescribed antibiotics is dependent on the arm of the model and has been taken from 3-year follow-up data in Cals et al. [9] described above. This study does not include a practice nurse plus CRP group and so these values have been assumed to be the same as GP plus CRP.

\section{Probabilities: Markov Model}

The transition probabilities used to populate the Markov model are shown in Table 2 [9]. The model replicates RTI rates from Cals et al. [9] over 28-day cycle lengths. The RTI rates are input as the number per person per year and then converted into 28-day probabilities in the model using the formula probability= $1-\exp$ (rate $\times$ time period) [12]. At baseline all 100 patients start in the RTI state, and in each cycle all patients that have not died have 28-day 
Table 1 Probabilities: decision tree

\section{Value in model (\%) Alpha Beta Distribution References}

in PSA

Antibiotics prescribed at index consultation

$\begin{array}{ll}\text { Current practice } & 59 \\ \text { GP plus CRP } & 39 \\ \text { Practice nurse plus CRP } & 4 \\ \text { GP plus CRP and communication training } & 35\end{array}$

$\begin{array}{rrrll}59 & 5,455 & 3,742 & \text { Beta } & \text { Huang [5], Little [7] } \\ 39 & 1,977 & 3,095 & \text { Beta } & \text { Huang [5], Little [7] } \\ 41 & 23 & 33 & \text { Beta } & \text { Cals [10] } \\ 35 & 897 & 1,636 & \text { Beta } & \text { Cals [6], Little [7] }\end{array}$

Antibiotics prescription within 28 days of index

$\begin{array}{llrrrc}\text { Current practice } & 38 & 349 & 571 & \text { Beta } & \text { Huang [5] } \\ \text { GP plus CRP } & 32 & 322 & 680 & \text { Beta } & \text { Huang [5] } \\ \text { Practice nurse plus CRP } & 46 & 26 & 30 & \text { Beta } & \text { Cals [10] } \\ \text { GP plus CRP and communication training } & 38 \% & 76 & 125 & \text { Beta } & \text { Cals [6] }\end{array}$

Antibiotics prescribed for subsequent incidents of RTIs after 28 days

$\begin{array}{llllll}\text { Current practice } & 36 & 63 & 113 & \text { Beta } & \text { Cals [9] } \\ \text { GP plus CRP } & 31 & 62 & 141 & \text { Beta } & \text { Cals [9] } \\ \text { Practice nurse plus CRP } & 31 & 62 & 141 & \text { Beta } & \text { Cals [9] } \\ \text { GP plus CRP and communication training } & 26 & 47 & 131 & \text { Beta } & \text { Cals [9] }\end{array}$

\begin{tabular}{lllllr}
\hline Adverse events & Value in model (\%) & Upper (\%) & Lower (\%) & Distribution in PSA & References \\
\hline Anaphylactic reaction to penicillin & 0.05 & 0.1 & 0.0025 & Beta & BNF [11] \\
Death due to anaphylactic shock & 10 & 20 & 5 & Beta & NICE [1] \\
Complications with antibiotics & 1.2 & 1.5 & 0.9 & Beta & NICE [1] \\
Complications no antibiotics & 4.7 & 5.9 & 3.6 & Beta & NICE [1]
\end{tabular}

BNF British National Formulary, CRP C-reactive protein, GP general practitioner, NICE National Institute for Health and Care Excellence, $P S A$ probabilistic sensitivity analysis, RTIs respiratory tract infections

transition probabilities for healthy and RTI applied. The health states of patients in preceding cycles are independent of and do not impact on the probability of patients being healthy or having an RTI in subsequent cycles.

As described above, the model assumes that the only way that a patient can die is following an antibiotic-related adverse event. This is captured in the decision tree only and hence no absorbing state of death has been included in the Markov model.

\section{Measuring Costs}

The Afinion ${ }^{\mathrm{TM}}$ Analyzer (Alere Ltd, Stockport, UK) device [13] is used as the model for the analysis in this study because it is widely used across Europe for CRP testing and also used in England for health assessments including glycated hemoglobin (HbA1c) POCT. The costs included in this model cover the incremental costs of the CRP test and the costs associated with managing an RTI in primary care in the 
Table 2 Probabilities in Markov model: RTIs per patient, per year

\begin{tabular}{llllll}
\hline Variable & Value in model & Upper & Lower & $\begin{array}{l}\text { Distribution } \\
\text { in PSA }\end{array}$ & References \\
\hline Current practice & 0.39 & 0.45 & 0.32 & Lognormal & Cals [9] \\
GP plus CRP & 0.34 & 0.40 & 0.28 & Lognormal & Cals [9] \\
Practice nurse plus CRP & 0.34 & 0.40 & 0.28 & Lognormal & Cals [9] \\
GP plus CRP and communication training & 0.33 & 0.36 & 0.29 & Lognormal & Cals [9] \\
\hline
\end{tabular}

$C R P$ C-reactive protein, GP general practitioner, $P S A$ probabilistic sensitivity analysis, $R T I s$ respiratory tract infections

NHS in England. Routine GP and hospital appointments for other conditions not related to an RTI are not included, since these are assumed to be common to all study arms and will not affect the incremental cost.

The cost of CRP includes the cost of one GP consultation and the cost per test of the CRP test material, machine depreciation and GP training for CRP. Values used in the model are shown in Table 3 [13]. In the GP plus CRP arm, the cost of a CRP test includes the cost per minute of GP time multiplied by the duration in minutes that it takes the GP to do a CRP test. This assumes that there is an opportunity cost associated with conducting a CRP test, in that the time a GP spends doing the CRP test is time that could be spent on other patient-related activities that could confer a different benefit. In the practice nurse plus CRP arm, the cost of CRP includes the cost per minute of a primary care nurse multiplied by the duration of a CRP test. The same assumptions relating to opportunity cost apply. The GP plus CRP and communication training arm includes the cost in minutes of GP time multiplied by the duration of the test plus the cost of the communication training. The cost of communication training is a oneoff cost per patient at baseline. In the current practice arm, the cost of a GP consultation only is included. The values in the model can easily be changed as further information becomes available.

At baseline, all patients incur the cost of a GP consultation and the cost of a CRP test and training, dependent on the CRP arm. In subsequent incidents of RTI, CRP is added as a weighted cost, the probability of which was taken from Cals et al. [9] since not all patients receive CRP in subsequent consultations.

The cost of antibiotics is a weighted cost calculated from Health and Social Care Information Centre data for 2013 on the prescription of antibiotics used in the treatment of RTI [14]. The cost of antibiotics per cycle is then the average cost per prescription multiplied by the number of patients prescribed antibiotics each cycle at the start of the cycle and within 28 days.

It is assumed that each RTI has an associated weighted cost to the health service per 28 days. The cost components currently included in the model for each RTI are assumed to be as follows (Table 3 [13-16], Table 4 [13, 17]):

- GP practice visit (number of visits, inclusive of the first visit for RTI symptoms);

- out of hours GP consultation (number of visits);

- outpatient attendance (number of attendances);

- hospital admission (number of admissions);

- chest X-ray (number of X-rays); 
Table 3 Costs

\begin{tabular}{|c|c|c|c|c|c|}
\hline & $\begin{array}{l}\text { Value in } \\
\text { model }\end{array}$ & Upper & Lower & $\begin{array}{l}\text { Distribution in } \\
\text { PSA }\end{array}$ & References \\
\hline \multicolumn{6}{|l|}{ Cost per CRP test only } \\
\hline Test material (reagent) & $£ 4.19$ & & & & Alere $[13]$ \\
\hline Depreciation of machine & $£ 1.34$ & & & & Alere $[13]$ \\
\hline Cost of GP training & $£ 0.40$ & & & & Alere $[13]$ \\
\hline Duration of test: GP (min) & 3 & 3.75 & 2.25 & Gamma & Alere $[13]$ \\
\hline Cost per minute: GP & $£ 3.80$ & & & & PSSRU [15] \\
\hline Duration of test: nurse (min) & 5 & 6.25 & 3.75 & Gamma & Alere $[13]$ \\
\hline Cost per minute: nurse & $£ 0.87$ & & & & PSSRU $[15]$ \\
\hline Cost per antibiotic prescription & $£ 3.11$ & $£ 3.88$ & $£ 2.33$ & Gamma & $\begin{array}{l}\text { Health and Social Care } \\
\text { Information Centre }[14]\end{array}$ \\
\hline $\begin{array}{l}\text { One-off cost per patient of } \\
\text { communication training }\end{array}$ & $£ 5.52$ & & & & Alere $[13]$ \\
\hline \multicolumn{6}{|l|}{ Unit costs } \\
\hline GP consultation & $£ 45.00$ & $£ 56.00$ & $£ 34.00$ & Gamma & PSSRU [15] \\
\hline GP out of hours & $£ 114.00$ & $£ 143.00$ & $£ 85.00$ & Gamma & PSSRU [15] \\
\hline Hospital outpatients & $£ 135.00$ & $£ 158.00$ & $£ 91.00$ & Gamma & PSSRU [15] \\
\hline Hospital admission & $£ 697.00$ & $£ 846.00$ & $£ 479.00$ & Gamma & PSSRU [15] \\
\hline Chest X-ray & $£ 122.00$ & $£ 137.00$ & $£ 74.00$ & Gamma & NHS Reference Costs [16] \\
\hline Blood & $£ 4.00$ & $£ 5.00$ & $£ 1.00$ & Gamma & NHS Reference Costs [16] \\
\hline Other (sputum, spirometry) & $£ 51.00$ & $£ 75.00$ & $£ 45.00$ & Gamma & NHS Reference Costs [16] \\
\hline
\end{tabular}

CRP C-reactive protein, GP general practitioner, NHS National Health Service, PSA probabilistic sensitivity analysis, PSSRU Personal Social Services Research Unit

- blood test (number of blood tests);

- other (number of sputum or spirometry tests).

Resource use (excluding hospital admissions) per patient per RTI incident was taken from the Cals et al. [18] economic evaluation of improving management of patients with acute cough by CRP point-of-care testing and communication training (IMPAC3T) at 28-day follow-up. Costs were converted to 2012/2013 prices using the hospital and community health services index [15]. The NICE draft guidance on pneumonia [17] found a significant difference in hospital admissions for CRP versus usual care. Hospital admissions were not included in the Cals et al. [18] analysis, but have been included in the model in light of this finding. There was no evidence for resource use for the practice nurse plus CRP arm; therefore, the same values have been used as for the GP plus 
Table 4 Average resource use per patient

\begin{tabular}{llll}
\hline Variable & $\begin{array}{l}\text { GP plus CRP or } \\
\text { nurse plus CRP }\end{array}$ & $\begin{array}{l}\text { GP plus CRP and } \\
\text { communication training }\end{array}$ & $\begin{array}{l}\text { Current } \\
\text { practice }\end{array}$ \\
\hline CRP test [13] & 0.04 & 0.04 & N/A \\
GP consultation [13] & 1.4 & 1.27 & 1.37 \\
GP out of hours [13] & 0.01 & 0.02 & 0.08 \\
Hospital outpatients [13] & 0.02 & 0.02 & 0 \\
Hospital admission [17] & 0.009 & 0.009 & 0.003 \\
Chest x-ray [13] & 0.05 & 0.09 & 0.07 \\
Blood [13] & 0.01 & 0.05 & 0.0 \\
Other (sputum, spirometry) [13] & 0.02 & 0.02 & 0.02 \\
\hline
\end{tabular}

$C R P$ C-reactive protein, GP general practitioner

CRP arm. Unit cost data were taken from standard published sources and published reports $[15,16]$.

\section{Measuring Outcomes}

The main outcome measure is QALYs; the number of antibiotics prescribed and the number of RTIs over 3 years were also calculated.

For the measurement of QALYs, each state in the Markov model is assigned a utility score and the total QALYs attributable to treatment depend on the number of patients in each state over the time horizon of the model. The QALYs for RTI health states are dependent on the arm of the model. Patients that experience an adverse event or complication have a utility decrement for that cycle. The utility scores associated with each state currently used in the model are listed in Table $5[1,8$, 18 , 19]. The following assumptions are made regarding the measurement of QALYs in the model:

- the utility score associated with day-to-day health is that of the general population;
- the utility score associated with RTI symptoms is the same for all four arms of the model;

- the utility decrement associated with adverse events and complications is the same for all four arms of the model;

- deaths from antibiotic-related adverse events are assumed to occur on the first day of each cycle; therefore the QALYs associated with each cycle in these states is zero;

- the duration of an RTI is determined by the arm of the model.

When patients are in the RTI state, it is assumed that they are healthy for all days that they do not have an RTI. The QALY for the RTI health state is hence calculated as 28 days, minus the duration of an RTI, times the daily utility score for being healthy plus the duration of an RTI multiplied by the daily utility score for an RTI.

\section{Measuring Cost-Effectiveness}

Cost-effectiveness is measured as the net monetary benefit (NMB) of each arm in the model to allow for ranking of the four different 
Table 5 QALYs

\begin{tabular}{lllll}
\hline & $\begin{array}{l}\text { Value in } \\
\text { model }\end{array}$ & $\begin{array}{l}\text { Standard } \\
\text { error }\end{array}$ & $\begin{array}{l}\text { Distribution in } \\
\text { PSA }\end{array}$ & References \\
\hline Utility scores & & & & Kind [19] \\
Utility score: health & 0.86 & 0.003949 & Beta & Oppong [8] \\
Utility score: RTI & 0.674 & 0.010138 & Beta & NICE [1] \\
Utility decrement: adverse event & -0.2 & & & \\
Duration of RTI (days) & & & Gamma & Cals [18] \\
Current practice & 9.7 & 0.56598 & Cals [18] \\
GP plus CRP & 9.1 & 0.591147 & Gamma & Cals [18] \\
Practice nurse plus CRP & 9.1 & 0.591147 & Gamma & Cals [18] \\
GP plus CRP and communication training & 11 & 0.637905 & Gamma & \\
\hline
\end{tabular}

CRP C-reactive protein, GP general practitioner, NICE National Institute for Health and Care Excellence, PSA probabilistic sensitivity analysis, $R T I$ respiratory tract infection

options, with the highest NMB being considered the most favorable option. NMB is calculated as the total QALYs, multiplied by the willingness to pay (WTP) for a QALY, minus the total cost.

The time horizon of the model is 3 years. An annual discount rate of $3.5 \%$ for future costs and benefits is used, applied as a discount rate per cycle of $0.26 \%$. All costs are calculated based on prices in 2012/2013, in UK pounds. The analytical perspective is the health service; any cost implications to social services, the individual, the family or employers are excluded.

Values for the total discounted cost and QALYs for each option are provided in Table 5 $[1,8,18,19]$.

\section{Sensitivity Analysis}

Uncertainty in costs and outcomes is investigated in the model with a probabilistic sensitivity analysis (PSA) [20] of 5,000 replications. A scatter plot of the cost- effectiveness plane showing incremental costs per 100 patients and the QALY gains per 100 patients of the three CRP test strategies compared with current practice, which is assumed to be at the origin on the costeffectiveness plane, is plotted for each replication. The probability that each of the four strategies has the highest $\mathrm{NMB}$ at various thresholds of WTP for a QALY-the costeffectiveness acceptability curve (CEAC) [21] is also plotted. Head-to-head comparisons of standard care compared to any options that are not dominated or eliminated by extended dominance are also reported.

The variables allowed to vary in the PSA, probability distributions and parameter values in the model are shown in Tables 1, 2, 3 and 4 and are chosen in line with the methodology set out in Briggs et al. [12]. One-way sensitivity analyses of key parameters in the model were also conducted.

The analysis was repeated for a 9-year time horizon (120 cycles of 28 days per cycle) to evaluate the impact on the results. 


\section{Compliance with Ethics Guidelines}

The analysis in this article is based on previously conducted studies and does not involve any new studies of human or animal subjects performed by any of the authors.

\section{RESULTS}

\section{Deterministic Analysis}

The results of the deterministic analysis are reported in Table 6. For the deterministic

Table 6 Results for 3 years per 100 patients

\begin{tabular}{lllll}
\hline Statistic & Current practice & GP plus CRP & Practice nurse plus CRP & $\begin{array}{l}\text { GP plus CRP and } \\
\text { communication }\end{array}$ \\
\hline Total cost (discounted) & $£ 18,081$ & $£ 18,039$ & $£ 17,401$ & $£ 18,431$ \\
QALYs (discounted) & 255.630 & 255.764 & 255.761 & 255.588 \\
Antibiotics prescribed (courses) & 184 & 136 & 167 & 137 \\
Infections & 217.89 & 202.97 & 202.97 & 199.98 \\
Per-patient NMB & $£ 50,945$ & $£ 50,972$ & $£ 50,978$ & $£ 50,933$ \\
$(£ 20,000$ per QALY) & & & & \\
\hline
\end{tabular}

$C R P$ C-reactive protein, $G P$ general practitioner, $N M B$ net monetary benefit, $Q A L Y$ quality-adjusted life year

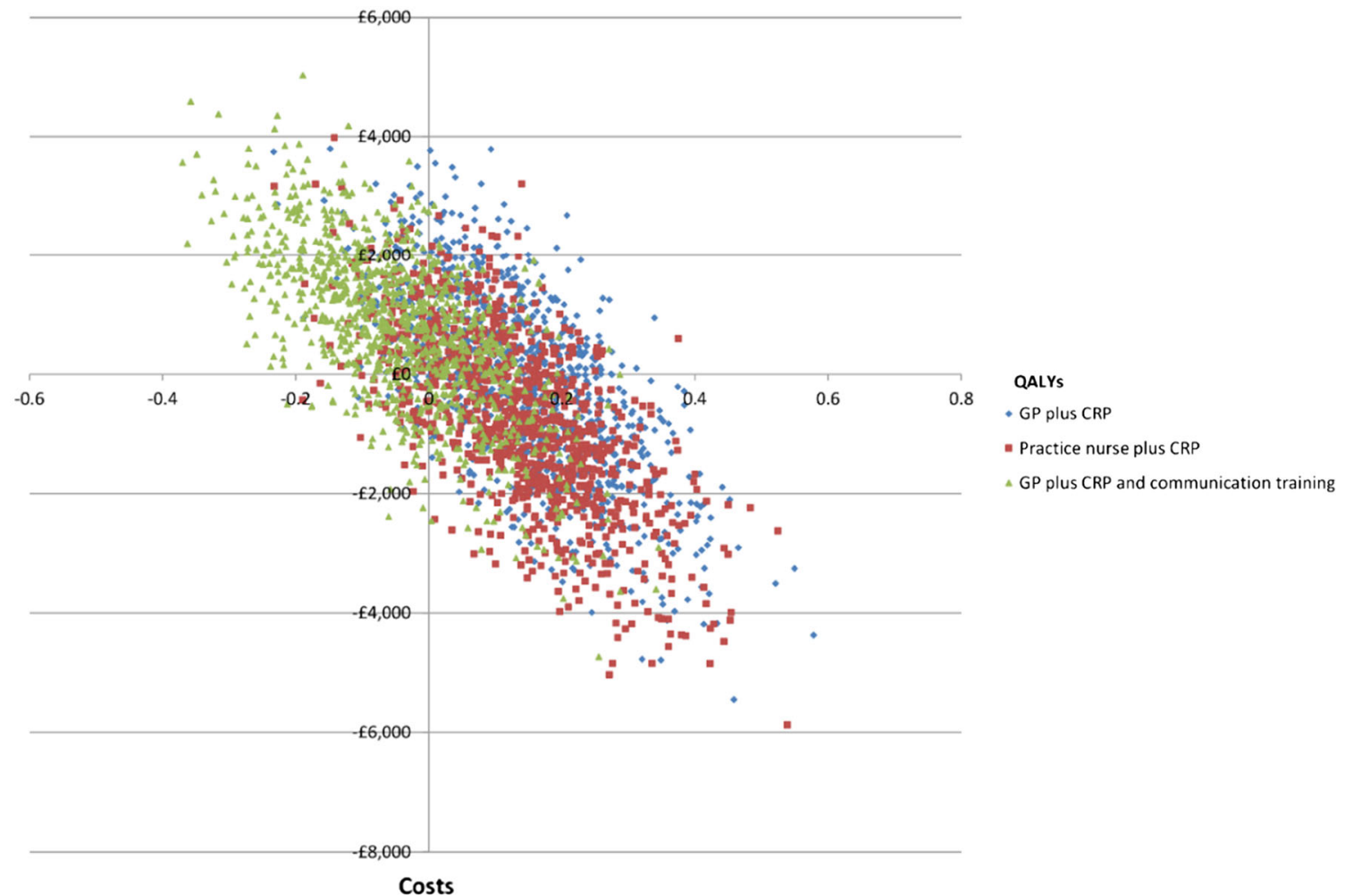

Fig. 3 Cost-effectiveness plane: current practice compared to GP plus CRP, practice nurse plus CRP, and GP plus CRP and communication training. Three-year time horizon, 100 patients and discounted costs and QALYs. 1,000 iterations. CRP C-reactive protein, GP general practitioner, $Q A L Y s$ quality-adjusted life years 
model, GP plus CRP test and practice nurse plus CRP test both dominate (cost less and result in more QALYs) compared to current practice over 3 years. The two strategies result in 0.13 additional QALYs per 100 patients (discounted) and cost $£ 42$ less per 100 patients (discounted) for GP plus CRP and $£ 680$ less per 100 patients (discounted) for practice nurse plus CRP. GP plus CRP test and communication training is dominated by current practice as it costs more and results in fewer QALYs. All three CRP arms result in fewer antibiotic prescriptions and infections over 3 years than current practice.

\section{Probabilistic Sensitivity Analysis}

The results for each of the simulations of the model compared to current practice are presented on a cost-effectiveness plane in Fig. 3.
The GP plus CRP test strategy is dominant (costs less and results in more QALYs) compared to current practice in $50 \%$ of simulations; in $65 \%$ of simulations the practice nurse plus CRP test strategy is dominant and in 19\% the GP plus CRP and communication training strategy is dominant.

A CEAC comparing the proportion of iterations of the PSA where each option has the highest NMB for a range of values of WTP for a QALY is presented in Fig. 4. The practice nurse plus CRP strategy has the highest proportion of iterations with the highest NMB across all values of WTP for a QALY, with values between $49 \%$ and $53 \%$.

Cost-effectiveness acceptability curves comparing current practice against GP plus CRP and practice nurse plus CRP are reported in Figs. 5 and 6, respectively. At a WTP of

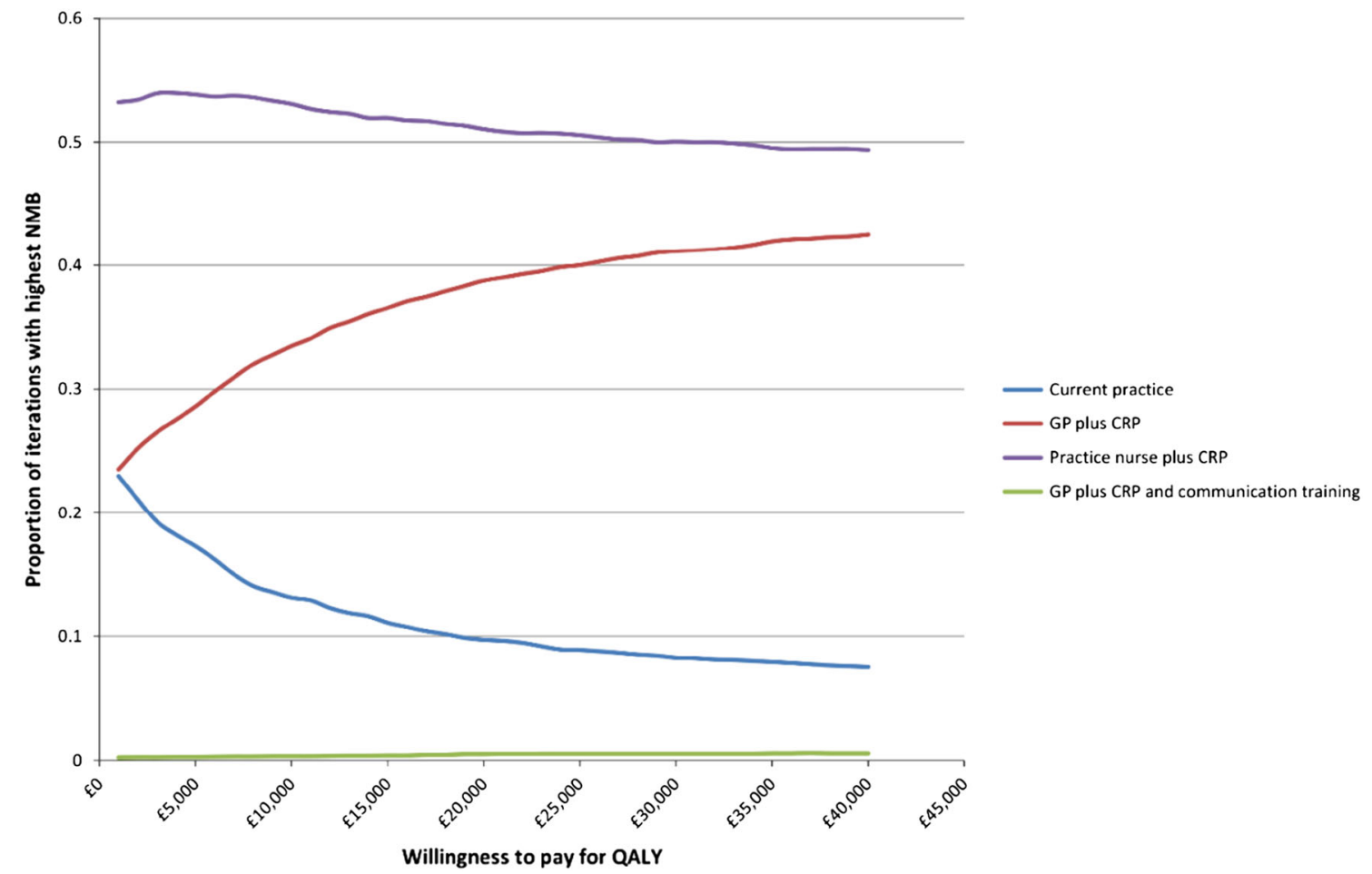

Fig. 4 Cost-effectiveness acceptability curve: current practice compared to GP plus CRP, practice nurse plus CRP, and GP plus CRP and communication training. Three-year time horizon, 100 patients and discounted

costs and QALYs. 5,000 iterations. CRP C-reactive protein, $G P$ general practitioner, $N M B$ net monetary benefit, $Q A L Y$ s quality-adjusted life years 
$£ 20,000$ per QALY, GP plus CRP has a higher NMB than current practice for $77 \%$ of iterations and practice nurse plus CRP has a higher NMB than current practice for $82 \%$ of iterations. The analysis for GP plus CRP and communication training was not presented given that it is dominated by the other three options.

The results of the one-way sensitivity analysis are presented in Table 7 . Changing most of the key parameters in the model has little impact on the conclusions, except for standardizing the length of RTI. If the length of RTI is standardized across all arms GP plus CRP and communication has a higher NMB than GP plus CRP and current practice, but not practice nurse plus CRP.

The results remain the same for the 9-year time horizon (see Fig. 7).

\section{DISCUSSION}

\section{Summary of Results}

Over a 3-year time horizon, two of the CRP test strategies, GP plus CRP test and practice nurse plus CRP test, have a higher NMB than current practice, although this difference is minimal. The additional cost per patient of the CRP test is outweighed by the associated cost savings and QALY increment associated with a reduction in infections in the long term. This result is mostly driven by the results of Cals et al. [9] which found that a point-of-care CRP test resulted in reduced risk of RTI per person per year compared to current practice, although the change was not significant.

Although communication training in addition to the GP CRP test also results in

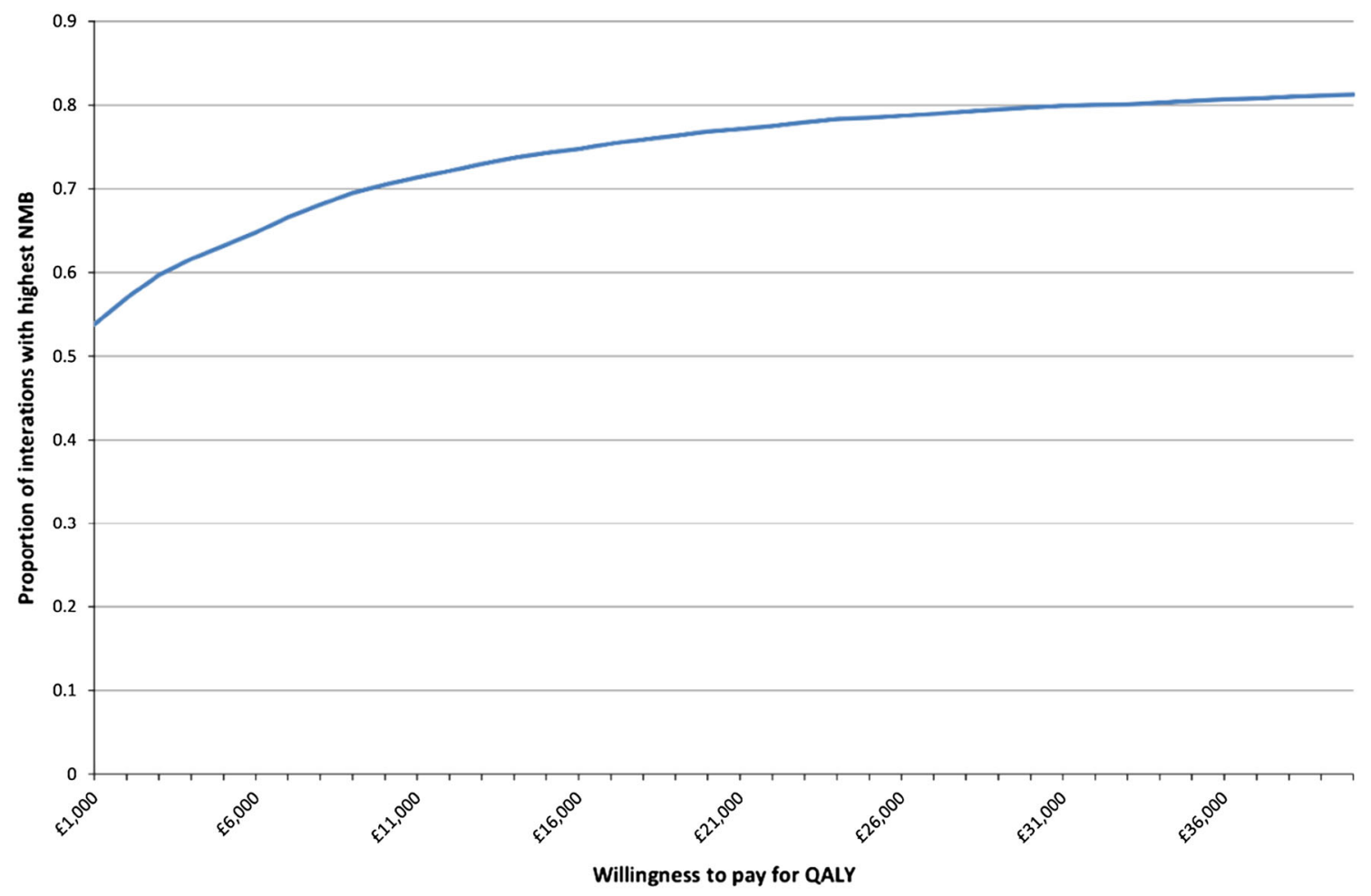

Fig. 5 Cost-effectiveness acceptability curve: current practice compared to GP plus CRP. Three-year time horizon, 100 patients and discounted costs and QALYs.
5,000 iterations. CRP C-reactive protein, $G P$ general practitioner, $N M B$ net monetary benefit, $Q A L Y s$ qualityadjusted life years 


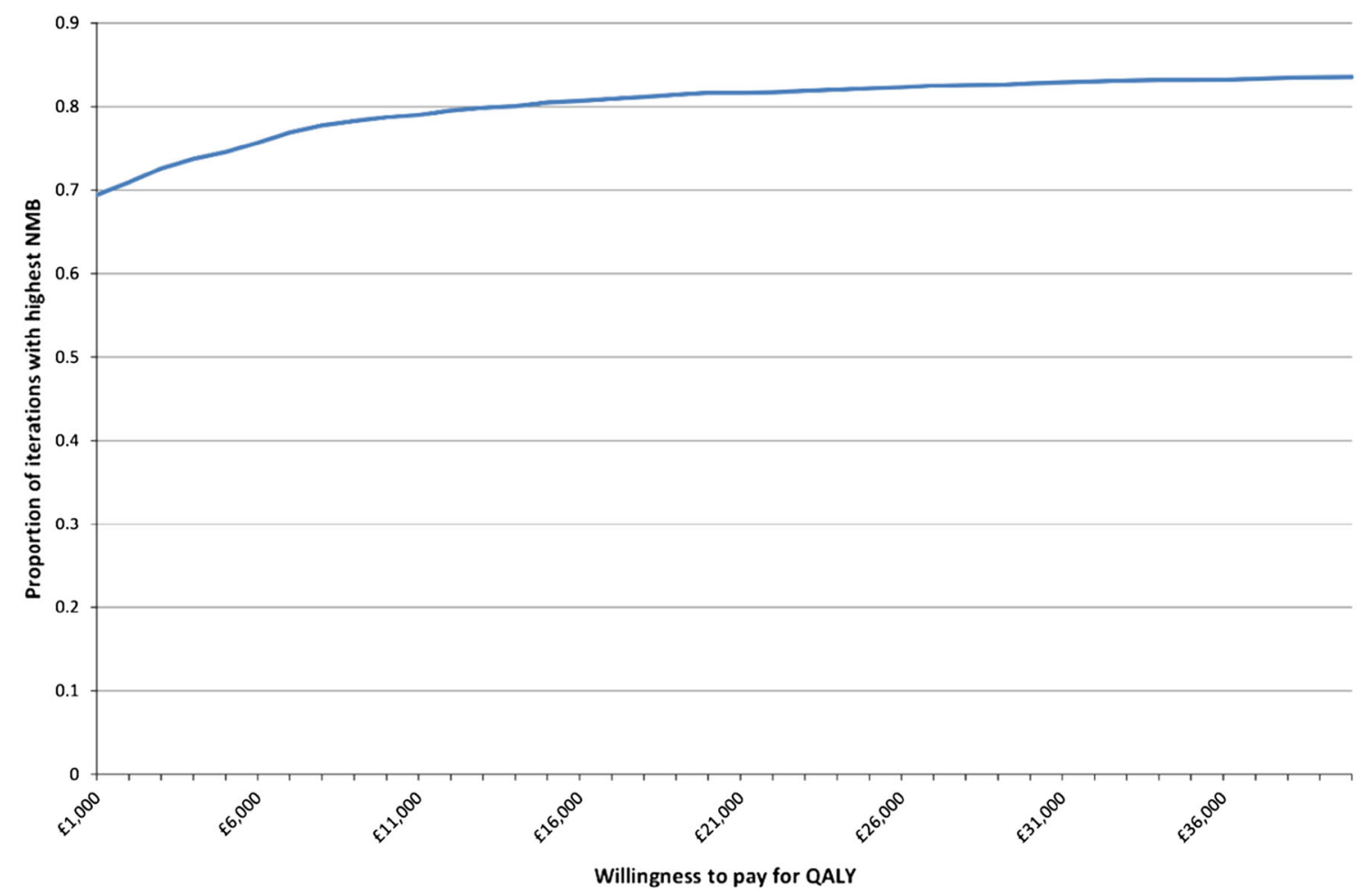

Fig. 6 Cost-effectiveness acceptability curve: current QALYs. 5,000 iterations. CRP C-reactive protein, $N M B$ practice compared to practice nurse plus CRP. Three-year net monetary benefit, $Q A L Y s$ quality-adjusted life years time horizon, 100 patients and discounted costs and

Table 7 One-way sensitivity analyses of net monetary benefit (NMB) per patient over 3 years at $£ 20,000$ per QALY

\begin{tabular}{lllll}
\hline Variable modified & $\begin{array}{l}\text { Current } \\
\text { practice }\end{array}$ & $\begin{array}{l}\text { GP plus } \\
\text { CRP }\end{array}$ & $\begin{array}{l}\text { Practice nurse } \\
\text { plus CRP }\end{array}$ & $\begin{array}{l}\text { GP plus CRP and } \\
\text { communication }\end{array}$ \\
\hline CRP test costs $50 \%$ more & $£ 50,945$ & $£ 50,970$ & $£ 50,975$ & $£ 50,936$ \\
CRP test costs 100\% more & $£ 50,945$ & $£ 50,967$ & $£ 50,972$ & $£ 50,933$ \\
CRP test takes twice as long & $£ 50,945$ & $£ 50,961$ & $£ 50,974$ & $£ 50,927$ \\
Antibiotics cost twice as much & $£ 50,940$ & $£ 50,968$ & $£ 50,973$ & $£ 50,935$ \\
Duration of RTI is 7 days & $£ 51,003$ & $£ 51,015$ & $£ 51,020$ & $£ 51,018$ \\
Duration of RTI is 11 days & $£ 50,917$ & $£ 50,934$ & $£ 50,940$ & $£ 50,939$ \\
Twice the risk of anaphylaxis & $£ 50,942$ & $£ 50,970$ & $£ 50,975$ & $£ 50,936$ \\
Twice the risk of complications with & $£ 50,944$ & $£ 50,972$ & $£ 50,978$ & $£ 50,938$ \\
antibiotics & & & & \\
Twice the risk of complications with no & $£ 50,943$ & $£ 50,970$ & $£ 50,976$ & $£ 50,937$ \\
antibiotics & & & & \\
Utility score of 1 if healthy & $£ 59,145$ & $£ 59,192$ & $£ 59,198$ & $£ 59,132$ \\
\hline
\end{tabular}


Table 7 continued

\begin{tabular}{lllll}
\hline Variable modified & $\begin{array}{l}\text { Current } \\
\text { practice }\end{array}$ & $\begin{array}{l}\text { GP plus } \\
\text { CRP }\end{array}$ & $\begin{array}{l}\text { Practice nurse } \\
\text { plus CRP }\end{array}$ & $\begin{array}{l}\text { GP plus CRP and } \\
\text { communication }\end{array}$ \\
\hline Utility score 0.1 higher for the duration of an RTI & $£ 51,058$ & $£ 51,071$ & $£ 51,077$ & $£ 51,056$ \\
Utility score 0.1 lower for the duration of an RTI & $£ 50,833$ & $£ 50,874$ & $£ 50,880$ & $£ 50,821$ \\
\hline
\end{tabular}

$C R P$ C-reactive protein, $G P$ general practitioner, $R T I$ respiratory tract infection

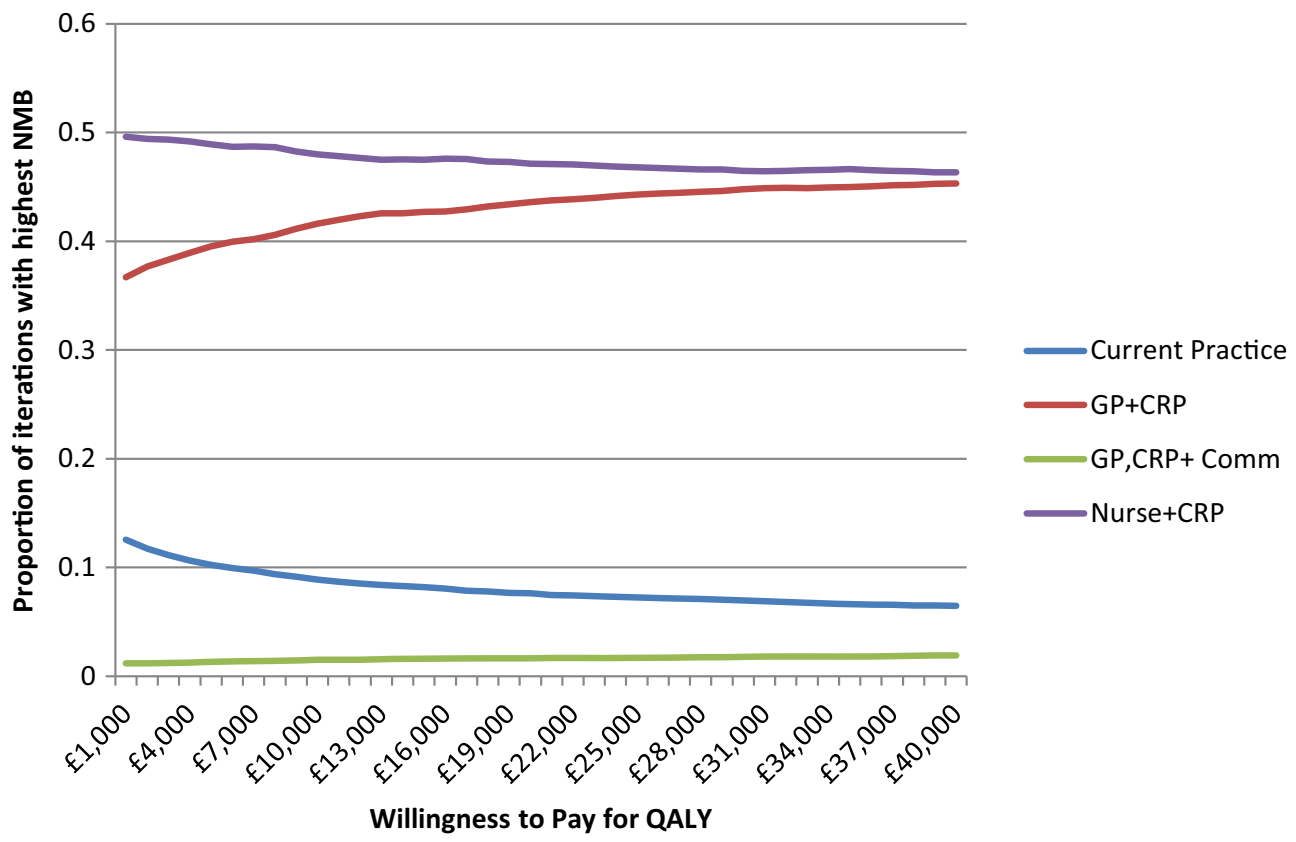

Fig. 7 Cost-effectiveness acceptability curve: current practice compared to GP plus CRP, practice nurse plus $\mathrm{CRP}$, and GP plus CRP and communication training-9-

reduced risk of infection and antibiotic prescribing, this is not sufficient to outweigh the additional cost of training. Two studies, Cals et al. [10] and Little et al. [7], also found that the duration of RTI symptoms is significantly longer for patients in communication training arms of trials, although only the results from Cals et al. [10] have been included in this model. The extended duration of symptoms is the likely explanation for why the GP plus CRP test plus communication training arm of the model also resulted in fewer QALYs than current practice, as shown by the results of the one-way year time horizon. CRP C-reactive protein, GP general practitioner, $N M B$ net monetary benefit, $Q A L Y s$ qualityadjusted life years

sensitivity analysis. This also suggests that if CRP testing were implemented in NHS England, the most cost-effective options would also be the least resource intensive to implement: practice nurses and/or GPs would only need training in using CRP tests, with questionable additional benefit being gained from more intensive communication training.

\section{Strengths and Weaknesses}

This is the first study to report the costeffectiveness of different strategies of CRP tests 
for RTI in primary care over a time horizon greater than 28 days; the model takes multiple factors into consideration and can be amended as further data become available.

However, the model has some limitations. Firstly, there are limited data on long-term antibiotic prescribing, health-care resource use and incidence of RTI for the point-of-care CRP strategy where the practice nurse conducts the CRP test followed by a consultation with the GP. Instead, the assumption was made that the results are the same as the GP conducting the CRP test and prescribing antibiotics for the missing variables. Given that the cost-effectiveness of the different strategies is strongly driven by these values, it reduces the strength of confidence in these results and the ability to validate the model using another data set.

Secondly, the 3-year follow-up results in Cals et al. [9] only capture the incidence of RTI where patients present to their GP with symptoms. If patients are discouraged from attending their GP because at their index appointment for RTI symptoms they were less likely to be prescribed antibiotics after having received a CRP test, they may perceive there is less value in attending the GP for subsequent RTIs. Hence, the reduced incidence of reported RTI may only be due to reduced primary care attendance, and not an actual reduction in RTI incidence. Although this is correctly reflected in the cost, as less attendance means reduced cost, there is the possibility that some of the disutility of having an RTI might be missing from the CRP arms of the model.

\section{CONCLUSION}

Over the 3-year period, two of the CRP test strategies, GP plus CRP test and practice nurse plus CRP test, are dominant over current practice. Further work should be carried out to determine whether any differences exist between the nurse plus CRP test strategy and GP plus CRP test strategy for the missing variables in this study. The data suggest that these strategies are associated with reduced risks of infection and antibiotic prescribing, in combination with increased QALYs, and therefore could be implemented costeffectively in primary care as a potential strategy to help reduce unnecessary antibiotic prescriptions. This would contribute to the ongoing national and international efforts to reduce antibiotic-resistant infections.

\section{ACKNOWLEDGMENTS}

Sponsorship and article processing charges for this study were funded by Alere. Editorial assistance was provided by Toby Galbraith, PhD, and Charlotte Simpson, PhD, of IMC Healthcare Communication Ltd (London, UK), funded by Alere. Rachael Hunter meets the International Committee of Medical Journal Editors (ICMJE) criteria for authorship for this manuscript, takes responsibility for the integrity of the work as a whole and has given final approval for the version to be published.

Conflict of interest. Rachael Hunter has received payment from Alere for analysis.

Compliance with ethics guidelines. The analysis in this article is based on previously conducted studies and does not involve any new studies of human or animal subjects performed by any of the authors.

Open Access. This article is distributed under the terms of the Creative Commons Attribution Noncommercial License which 
permits any noncommercial use, distribution, and reproduction in any medium, provided the original author(s) and the source are credited.

\section{REFERENCES}

1. Respiratory tract infections-antibiotic prescribing. NICE clinical guideline 69. NICE; London, UK; 2008. Available from: http://www.nice.org.uk/ guidance/cg69/. Accessed 1 June 2014.

2. Public Health England: English surveillance programme for antimicrobial utilisation and resistance (ESPAUR). Available from: https://www. gov.uk/government/uploads/system/uploads/attach ment_data/file/362374/ESPAUR_Report_2014__3_. pdf. Accessed 1 June 2014.

3. Butler C, Hood K, Verheij $\mathrm{T}$, et al. Variation in antibiotic prescribing and its impact on recovery in patients with acute cough in primary care: prospective study in 13 countries. BMJ. 2009;338:b2242.

4. Department of Health and Department for Environment, Food \& Rural Affairs: UK Five Year Antimicrobial Resistance Strategy 2013 to 2018. Available from: https://www.gov.uk/government/ uploads/system/uploads/attachment_data/file/2440 58/20130902_UK_5_year_AMR_strategy.pdf. Accessed 1 June 2014.

5. Huang Y, Chen R, Wu T, et al. Association between point-of-care CRP testing and antibiotic prescribing in respiratory tract infections: a systematic review and meta-analysis of primary care studies. Br J Gen Pract. 2013;63(616):e787-94.

6. Cals JW, Butler CC, Hopstaken RM, Hood K, Dinant GJ. Effect of point of care testing for $\mathrm{C}$ reactive protein and training in communication skills on antibiotic use in lower respiratory tract infections: cluster randomised trial. BMJ. 2009;338:b1374.

7. Little P, Stuart B, Francis N, et al. Effects of internetbased training on antibiotic prescribing rates for acute respiratory-tract infections: a multinational, cluster, randomised, factorial, controlled trial. Lancet. 2013;382(9899):1175-82.

8. Oppong R, Jit M, Smith RD, et al. Cost-effectiveness of point-of-care C-reactive protein testing to inform antibiotic prescribing decisions. Br J Gen Pract. 2013;63(612):e465-71.

9. Cals JW, de Bock L, Beckers PJ, et al. Enhanced communication skills and C-reactive protein pointof-care testing for respiratory tract infection: 3.5- year follow-up of a cluster randomized trial. Ann Fam Med. 2013;11(2):157-64.

10. Cals JW, Schot MJ, de Jong SA, Dinant GJ, Hopstaken RM. Point-of-care C-reactive protein testing and antibiotic prescribing for respiratory tract infections: a randomized controlled trial. Ann Fam Med. 2010;8(2):124-33.

11. Royal Pharmaceutical Society of Great Britain. British National Formulary (BNF) 67. BMJ Publishing Group Ltd and Royal Pharmaceutical Society; 2014.

12. Briggs A, Claxton $K$, Sculpher $M$. Decision modelling for health economic evaluation. Oxford: Oxford University Press; 2011. ISBN 978-019-852662-9.

13. Alere: Afinion ${ }^{\mathrm{TM}}$ AS100 Analyzer and tests. Available from: http://www.alere.co.uk/search/?q= afinion. Accessed 1 June 2014.

14. Health and Social Care Information Centre: Prescription Cost Analysis, England-2013. Available from http://www.hscic.gov.uk/catalogue/ PUB13887. Accessed 1 June 2014.

15. Curtis L. Unit costs of health and social care 2013. Personal social services research unit (PSSRU). Canterbury: University of Kent; 2014.

16. NHS reference costs: financial year 2012 to 2013. Department of Health; 2014.

17. Pneumonia: diagnosis and management of community- and hospital-acquired pneumonia in adults. NICE; London, UK; 2014. Draft guideline available from: http://www.nice.org.uk/guidance/ gid-cgwave0607/resources/pneumonia-guidelineconsultation-nice-guideline2. Accessed 1 June 2014.

18. Cals JW, Ament AJ, Hood K, et al. C-reactive protein point of care testing and physician communication skills training for lower respiratory tract infections in general practice: economic evaluation of a cluster randomized trial. J Eval Clin Pract. 2011;17(6):1059-69.

19. Kind P, Hardman G, Macran S. UK Population Norms for EQ-5D. Discussion paper 172. Centre for Health Economics, University of York; 1999.

20. Claxton K, Sculpher M, McCabe C, et al. Probabilistic sensitivity analysis for NICE technology assessment: not an optional extra. Health Econ. 2005;14:339-47.

21. Fenwick E, Claxton K, Sculpher M. Representing uncertainty: the role of cost-effectiveness acceptability curves. Health Econ. 2001;10:779-87. 